\title{
THE FLOW OF A VISCOUS COMPRESSIBLE FLUID THROUGH A VERY NARROW GAP*
}

\author{
J. D. COLE, H. B. KELLER AND P. G. SAFFMAN $\dagger$
}

1. Introduction. The effect of compressibility on the pressure distribution in the narrow gap between a rotating cylinder and a plane in a viscous fluid was studied by Taylor and Saffman [1] during an investigation of the centripetal pump effect discovered by Reiner [2]. With the usual assumptions of lubrication theory and the further assumption of an isothermal film which satisfies Boyle's law, the Navier-Stokes equations yield an equation for the pressure

$$
\frac{d}{d \phi}\left(\frac{p}{p_{0}}\right)=\beta \cos ^{2} \phi-\alpha\left(\frac{p_{0}}{p}\right) \cos ^{4} \phi .
$$

The independent variable $\phi$ is defined by

$$
x=(2 R h)^{1 / 2} \tan \phi,
$$

where $R$ is the radius of the cylinder and $h$ is the gap width as shown in Fig. 1. If $\omega$ is the angular speed of the cylinder (positive counterclockwise) and $U \equiv R \omega$, then the parameters $\alpha$ and $\beta$ are defined by

$$
\alpha \equiv \frac{12 \mu F}{p_{0} \rho_{0}}\left(\frac{2 R}{h^{5}}\right)^{1 / 2}, \quad \beta \equiv \frac{6 \mu U}{p_{0}}\left(\frac{2 R}{h^{3}}\right)^{1 / 2} .
$$

Here $\mu$ is the viscosity coefficient of the fluid, $F$ is the mass flux per unit span through the film, and $p_{0}$ and $\rho_{0}$ are the pressure and density at $|x|=\infty$. Thus we have assumed that the pressure satisfies

$$
p(-\pi / 2)=p(\pi / 2)=p_{0} .
$$

The vertical thrust, $T$, on the bearing is approximately

$$
T=\int\left(p-p_{0}\right) d x=p_{0}(2 R h)^{1 / 2} \int_{-(1 / 2) \pi}^{(1 / 2) \pi}\left(p / p_{0}-1\right) \sec ^{2} \phi d \phi .
$$

There is also a contribution from the viscous stresses which can be shown to be smaller by a factor of order $h / R$.

With but slight modifications the above formulation is adequate for describing other configurations of interest. For instance, if lubricant is forced through the gap by a pressure gradient, say with pressure $p_{0}$ on the entrance side $(x<0)$ and pressure $p_{2}<p_{0}$ on the exit side $(x>0)$, we

* Received by the editors September 7, 1966.

i California Institute of Technology, Pasadena, California. This research was supported in part by the National Science Foundation under Grant GP 4597. 


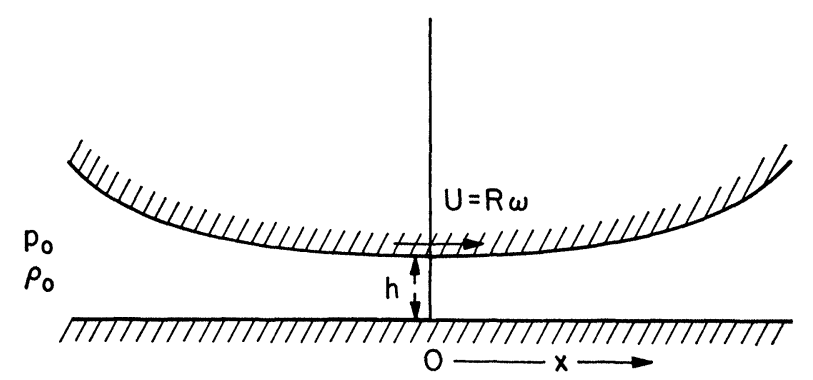

Fig. 1. Sketch of gap between rotating cylinder and fixed plane

need only replace the boundary conditions (3) by

$$
p(-\pi / 2)=p_{0}, \quad p(\pi / 2)=p_{2} .
$$

If the cylinder does not rotate, then $\beta=0$, (1) simplifies and is easily integrated; see $\$ 4$.

In general, the parameter $\beta$ is given and the problem is to find $p(\phi)$ and $\alpha$ such that (1) and (3) or (1) and (5) are satisfied. We call the former problem the case of free flow and the latter the case of forced flow. The free flow case is studied rather thoroughly, using ordinary perturbation theory for $\beta \ll 1$, using singular perturbations (or boundary layer expansions) for $\beta \gg 1$ in $\S 2$. The region of $\beta=O(1)$ and the accuracy of the expansions is studied by numerical solutions in $\S 3$. The forced flow case is briefly examined and we indicate how the free flow results apply to it in $\$ 4$.

2. Free flow. For $\beta$ small the perturbation problem for (1) with the boundary condition (3) is regular. For $\beta$ small the solution is expressed as a power series in $\beta$. Due to the extra boundary condition in (3) the parameter $\alpha$ is free and must also be expressed by a series expansion in $\beta$. The results to second order are:

$$
\begin{aligned}
\frac{p(\phi)}{p_{0}} & =1-\frac{\beta}{3} \sin \phi \cos ^{3} \phi+\frac{\beta^{2}}{18} \cos ^{8} \phi+O\left(\beta^{3}\right), \\
\alpha & =\frac{4}{3} \beta+O\left(\beta^{3}\right), \\
\frac{T}{p_{0} \sqrt{2 R h}} & =\frac{5 \pi}{288} \beta^{2}+O\left(\beta^{3}\right) .
\end{aligned}
$$

To first order in $\beta$ this is just the well-known incompressible limit solution which is odd about $x=0$ and has no thrust. The second order correction yields a nonzero thrust but does not alter the mass flux $\alpha$.

The solution for the case $\beta \gg 1$ is of some physical interest as it bears on the circumstance of a very narrow gap and a large thrust, a problem dominated by compressibility. The perturbation problem turns out to be singular 
and to have some interesting features. It is convenient to use the periodicity (period $\pi$ ) of the solution of (1) under the boundary conditions (3) to consider the problem in $0<\phi<\pi$. Let us rewrite the problem in terms of the independent variable $\theta=\phi-\pi / 2$ and a new dependent variable $y(\theta)=p / p_{1}$, where $p_{1}$ is the pressure at $x=0$. Then we have the equation

$$
y \frac{d y}{d \theta}=y B \sin ^{2} \theta-A \sin ^{4} \theta \text { for } \frac{\pi}{2}<\theta<\frac{\pi}{2},
$$

where

$$
B=\left(\frac{p_{0}}{p_{1}}\right) \beta, \quad A=\left(\frac{p_{0}}{p_{1}}\right)^{2} \alpha .
$$

We now study the solution of ( 7$)$ in the limit $B \rightarrow \infty$, regarding $B$ as given. The parameter $A=A(B)$ is to be determined by the boundary conditions

$$
y(\pi / 2)=y(-\pi / 2)=1,
$$

and the value of $p_{0} / p_{1}$ follows from

$$
y(0)=p_{0} / p_{1} .
$$

In this problem two asymptotic expansions with different regions of validity are needed. The first asymptotic expansion is associated with the limit process $B \rightarrow \infty, \theta$ fixed, the "outer" expansion. It can be seen from the form of the outer expansion below that it is not valid near $\theta=0$. A local expansion must be constructed near $\theta=0$ and these two expansions must be matched. The concepts developed in this process turn out to be useful also for general considerations of numerical analysis of the problem. Some details are now given.

The limit process expansion $(B \rightarrow \infty, \theta$ fixed) can be expressed as a simple power series:

$$
\begin{aligned}
y(\theta, B) & =y_{0}(\theta)+\frac{1}{B} y_{1}(\theta)+\frac{1}{B^{2}} y_{2}(\theta)+\frac{1}{B^{3}} y_{3}(\theta)+\cdots, \\
\frac{A(B)}{B} & =A_{0}+\frac{1}{B} A_{1}+\frac{1}{B^{2}} A_{2}+\frac{1}{B^{3}} A_{3}+\cdots
\end{aligned}
$$

On substituting in (7), equating coefficients of inverse powers of $B$ and applying the boundary condition (9), we obtain after some algebra:

$$
\begin{aligned}
y=\sin ^{2} \theta+\frac{2}{B} \sin \theta \cos \theta & +\frac{6}{B^{2}} \cos ^{2} \theta+\frac{16}{B^{3}} \frac{\cos \theta}{\sin \theta}\left(\cos ^{2} \theta-\sin ^{2} \theta\right) \\
& +\frac{24 \cos ^{2} \theta}{B^{4} \sin ^{2} \theta}\left(\cos ^{2} \theta-\frac{11}{2} \sin ^{2} \theta\right)+\cdots,
\end{aligned}
$$

(14) $A=B+\frac{2}{B}-\frac{16}{B^{3}}+\cdots$. 
The dominant terms of the expansions (13) and (14) are calculated from the right-hand side of $(7)$, the derivative term being small. This leads to nonuniform behavior of the expansion for $y$ in the neighborhood of $\theta=0$. The second term of the expansion (13) is small compared to the first only if

$$
\frac{1}{B \theta} \ll 1 \text {. }
$$

This difficulty shows up explicitly also in the fourth term of (13) which has a singularity.

In order to construct an expansion which is valid near $\theta=0$ variables must be chosen which remain fixed as $(\theta \rightarrow 0, y \rightarrow 0, B \rightarrow \infty)$ and in such a way that a distinguished first approximation equation results. This differential equation must include the derivative term and in this case an approximation to each other term so that the inner expansion can be matched to the outer expansion (13). A suitable choice of variables is

$$
\tilde{\theta}=B \theta, \quad \tilde{y}=B^{2} y,
$$

and the inner expansion, valid near $\theta=0$, has the form

$$
\tilde{y}(\tilde{\theta}, B)=\tilde{y}_{0}(\tilde{\theta})+f_{1}(B) \tilde{y}_{1}(\tilde{\theta})+f_{2}(B) \tilde{y}_{2}(\tilde{\theta})+\cdots,
$$

where $f_{i+1}(B) / f_{i}(B) \rightarrow 0$ as $B \rightarrow \infty$. The leading term satisfies the distinguished equation

$$
\tilde{y}_{0} \frac{d \tilde{y}_{0}}{d \tilde{\theta}}=\tilde{y}_{0} \tilde{\theta}^{2}-\tilde{\theta}^{4}
$$

The equations for $\tilde{y}_{1}, \tilde{y}_{2}$, etc., can be found and are linear. The matching of the two expansions (13) and (16) is expressed by an intermediate class of limits in which $B \rightarrow \infty, \theta_{\eta}=\theta / \eta(B)$ is fixed, where $\eta(B)$ is such that $\eta \rightarrow 0$ but $B \eta \rightarrow \infty$. Thus in the intermediate limit $\theta=\eta \theta_{\eta} \rightarrow 0, \tilde{\theta}=B \eta \theta_{\eta} \rightarrow \infty$. The outer solution near $\theta=0$ agrees with the inner near $\tilde{\theta}=\infty$. Under the intermediate limit the two expansions (16) and (13) must agree identically and this provides a way of choosing the correct integral curve for (17).

We have

$$
\begin{aligned}
\lim _{\substack{B \rightarrow \infty \\
\theta_{\eta} \text { fixed }}}\left\{\tilde{y}_{0}\left(B \eta \theta_{\eta}\right)+\right. & f_{1}(B) \tilde{y}_{1}\left(B \eta \theta_{\eta}\right)+\cdots \\
& \left.-B^{2}\left[\eta^{2} \theta_{\eta}{ }^{2}+\frac{2}{B} \eta \theta_{\eta}+\frac{6}{B^{2}}+\frac{16}{B^{3} \eta \theta_{\eta}}+\cdots\right]\right\}=0 .
\end{aligned}
$$

Neglecting the various smaller terms the matching condition yields a boundary condition for (17) which can be written again in terms of $\tilde{\theta}$ : 


$$
\lim _{\tilde{\theta} \rightarrow \pm \infty} \tilde{y}_{0}(\tilde{\theta})=\tilde{\theta}^{2}+2 \tilde{\theta}+6+\frac{16}{\tilde{\theta}}+O\left(\frac{1}{\tilde{\theta}^{2}}\right) .
$$

Thus to complete the determination of the leading term of the complete solution, we need to show that the solution of (17) subject to (18) exists, and to calculate $\tilde{y}_{0}(0)$.

For physical reasons only solutions with $y>0$ are relevant, and we restrict attention therefore to the upper half of the $\tilde{y}-\tilde{\theta}$ plane (see Fig. $2)$. The following facts are relevant. Positive and negative values of $d \tilde{y}_{0} / d \tilde{\theta}$ are separated by the parabola $\tilde{y}_{0}=\tilde{\theta}^{2}$. If in fact the boundary condition (18) determines a unique solution of (17), it follows that

$$
\frac{p_{0}}{p_{1}} \equiv y(0)=\frac{1}{B^{2}} \tilde{y}(0)+o\left(\frac{1}{B^{2}}\right) .
$$

Equation (17) is free of singularities away from the axes and thus one and only one solution passes through each point of $\tilde{y}-\tilde{\theta}$ plane. As $\tilde{y}_{0} \rightarrow+\infty$, it is found by direct substitution that the asymptotic development (18) defines a solution, but we find that in addition there are solutions for $\tilde{\theta} \rightarrow+\infty$ such that

$$
\begin{aligned}
\tilde{y}_{0} \sim & \frac{\tilde{\theta}^{3}}{3}-\frac{3 \tilde{\theta}^{2}}{2}-\frac{27 \tilde{\theta}}{2}+k+O\left(\frac{1}{\tilde{\theta}}\right) \\
& +\log \tilde{\theta}\left[-\frac{27^{2}}{4}+O\left(\frac{1}{\tilde{\theta}}\right)\right] \\
& +O\left[\frac{(\log \tilde{\theta})}{\tilde{\theta}}\right]^{2},
\end{aligned}
$$

where $k$ is arbitrary. In (20), the unspecified terms are uniquely determined when $k$ is given. The development (20) does not apply for negative $\tilde{\theta}$, but (18) is still a possibility. In fact, as $\tilde{\theta}$ decreases from zero, all solutions (with positive $\tilde{y}_{0}(0)$ ) decrease until they cross $\tilde{y}=\tilde{\theta}^{2}$ after which they increase as $\tilde{\theta}$ decreases since $d \tilde{y} / d \tilde{\theta}$ has changed sign. Thus all solutions of (17) as $\tilde{\theta} \rightarrow-\infty$ have the same asymptotic behavior, namely that given by (18), and hence different solutions differ by transcendentally small terms as $\tilde{\theta} \rightarrow-\infty$.

Consider now a solution of (17) which starts from some given value of $\tilde{y}_{0}(0)$. For positive $\tilde{\theta}$, there are three possibilities: (i) The solution crosses the parabola $\tilde{y}_{0}=\tilde{\theta}^{2}$ after which $d \tilde{y} / d \tilde{\theta}$ goes negative and $\tilde{y}_{0}$ eventually crosses the $\tilde{\theta}$ axis. (ii) The solution increases monotonically and eventually asymptotes $\tilde{y}_{0}=\frac{1}{3} \tilde{\theta}^{3}$. (iii) The solution grows monotonically but asymptotes $\tilde{y}_{0}=\tilde{\theta}^{2}$. Since solutions cannot cross, the three possibilities separate the $\tilde{y}_{0}$ axis into three intervals, and the intervals defined by (i) and (ii) are open and are separated by points which lead to solutions corresponding 
to (iii). Now consider $\tilde{y}_{0}+\delta \tilde{y}$, where $\tilde{y}_{0}$ is a solution which asymptotes (18), and $\delta \tilde{y}$ is infinitesimal. Substituting in the equation and neglecting squares of $\delta \tilde{y}$, we find that

$$
\frac{d}{d \tilde{\theta}}(\delta \tilde{y}) \sim \delta \tilde{y}, \quad \text { i.e., } \delta \tilde{y} \propto e^{\tilde{\theta}} .
$$

Thus for $\tilde{\theta}>0$, departures from $\tilde{y}_{0}$ grow exponentially, and there is only one solution which behaves asymptotically like (18). This shows that the matching defines a unique solution. On the other hand, for $\tilde{\theta}<0$, the departure from $\tilde{y}_{0}$ decays exponentially and solutions accumulate on the asymptote (18) for negative $\tilde{\theta}$. Thus for negative $\tilde{\theta}$, all the solutions have the same asymptotic behavior.

The properties of solution (iii) show that backward integration should be employed for numerical evaluation of $\tilde{y}_{0}$. Such a calculation was done by K. W. Jacob starting with the asymptotic value in (18) for $\tilde{\theta}=100$ and integrating (17) step by step (via Runge-Kutta) towards smaller values of $\tilde{\theta}$. The results are plotted in Fig. 2. It was found, using a stepsize of $\Delta x=0.2$, that

$$
\tilde{y}_{0}(0)=20.63 \text {. }
$$

The next term in the inner expansion is of interest in order to estimate the error. Substituting (16) into the differential equation, we find that the correct choice is $f_{1}(B)=B^{-2}$ and

$$
\frac{d \tilde{y}_{1}}{d \tilde{\theta}}-\frac{\tilde{\theta}^{4}}{\tilde{y}_{0}^{2}} \tilde{y}_{1}=-\frac{\tilde{\theta}^{4}}{3}+\frac{2}{3} \frac{\tilde{\theta}^{6}}{\tilde{y}_{0}}-\frac{2 \tilde{\theta}^{4}}{\tilde{y}_{0}} .
$$

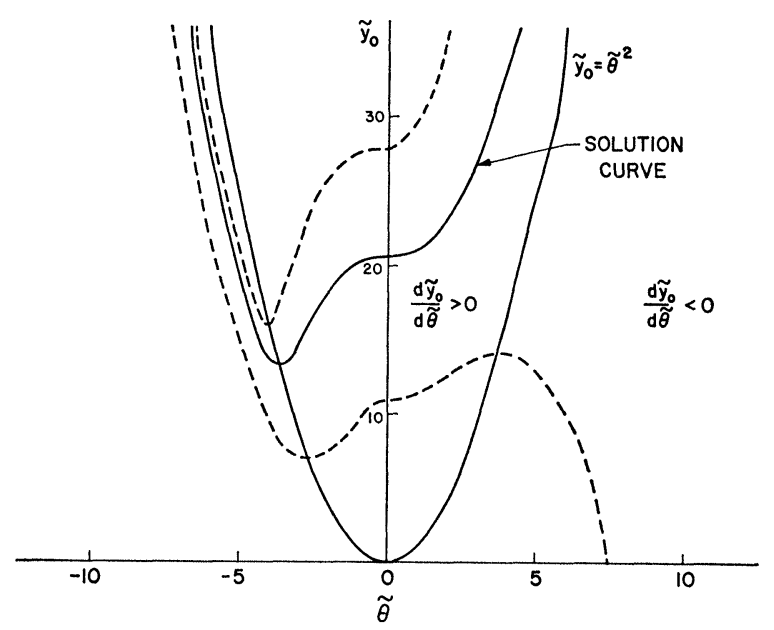

FIG. 2. Sketch of solution curves of (17) 
FLOW OF VISCOUS COMPRESSIBLE FLUID THROUGH A NARROW GAP 611

The matching requirement is found after reduction to imply

$$
\tilde{y}(\tilde{\theta})=-\frac{\tilde{\theta}^{4}}{3}-\frac{4 \tilde{\theta}^{3}}{3}-6 \tilde{\theta}^{2}-\frac{112 \tilde{\theta}}{3}+24+\cdots \quad \text { as } \quad \tilde{\theta} \rightarrow \pm \infty .
$$

Equation (23) is linear in $\tilde{y}_{1}$ and the discussion is straightforward. Keeping in mind that $\tilde{y} \sim \tilde{\theta}^{2}$, we see that all the solutions asymptote (24) as $\tilde{\theta} \rightarrow-\infty$ but that the solutions are proportional to $\pm e^{\tilde{\theta}}$ as $\tilde{\theta} \rightarrow+\infty$, except for one solution for a particular value of $\tilde{y}_{1}(0)$ which asymptotes (24). This is the solution we require. A crude estimate based on graphical integration of (23) gives

$$
\tilde{y}_{1}(0)=-600 .
$$

Thus the second term in the inner expansion is small compared with the first only if $B \gg 5$.

To summarize, we have the asymptotic expansions

$$
\begin{aligned}
& y=\sin ^{2} \theta+\frac{2}{B} \sin \theta \cos \theta+\frac{6}{B^{2}} \cos ^{2} \theta+O\left(\frac{1}{B^{3}}\right) \text { for } \theta \gg B^{-1}, \\
& y=\frac{1}{B^{2}} \tilde{y}_{0}(B \theta)+O\left(\frac{1}{B^{4}}\right) \text { for }|\theta| \ll B^{-1},
\end{aligned}
$$

where $\tilde{y}_{0}$ is plotted in Fig. 2 and has the asymptotic development (18), and $\tilde{y}_{0}(0)=20.63$.

A composite expansion correct to $O\left(B^{-2}\right)$ throughout the whole range can be constructed by inspection. It is

$$
\begin{aligned}
y(\theta)=\sin ^{2} \theta+\frac{2}{B} \sin \theta \cos \theta+\frac{6}{B^{2}} \cos ^{2} \theta+\frac{1}{B^{2}} \tilde{y}_{0}(B \theta) & \\
& -\left(\theta^{2}+\frac{2 \theta}{B}+\frac{6}{B^{2}}\right),
\end{aligned}
$$

the difference between $y(\theta)$ and (26) being $O\left(B^{-3}\right)$ for $\theta \gg B^{-1}$ and $\theta \ll B^{-1}$.

The pressure $p$ in the gap is given by

$$
p=p_{0} \frac{y}{y(0)},
$$

and the parameter $B$ is given in terms of the physical parameter $\beta$ by the equation

$$
\frac{B}{y(0)}=\beta \text {. }
$$

Thus

$$
B \sim(20.63 \beta)^{1 / 3},
$$


and the requirement $B \gg 5$ would require $\beta \gg 500$ at the least. Thus the asymptotic development requires very large values of the physical parameter $\beta$.

For the thrust on the bearing, it follows from (4) that

$$
\frac{T}{p_{0}(2 R h)^{1 / 2}}=\frac{1}{y(0)} \int_{-\pi / 2}^{\pi / 2}[y(\theta)-y(0)] \csc ^{2} \theta d \theta .
$$

On substituting the composite expansion (27) it is found that

$$
\frac{T}{p_{0}(2 R h)^{1 / 2}}=\frac{\pi B^{2}}{\tilde{y}_{0}(0)}\left[1+\frac{1}{B} \int_{-\infty}^{\infty}\left[\left(\tilde{y}_{0} \tilde{\theta}\right)-\tilde{y}_{0}(0)-\tilde{\theta}^{2}\right] d \tilde{\theta}+O\left(\frac{1}{B^{2}}\right)\right] .
$$

The coefficient of $B^{-1}$ was roughly estimated by graphical integration to be about -10 . It is noteworthy that the vicinity of $\theta=0$, where the relative value of $y$ is $O\left(B^{-2}\right)$, contributes a term of relative order $B^{-1}$ to the thrust.

3. Numerical solutions for free flow. The previous expansions, (6) valid for $\beta \ll 1$ and (26) valid for $B \gg 5$ or $\beta \gg 500$, leave a rather large range of parameter values for which no adequate approximation is known. To fill this gap and at the same time check on the accuracy of the approximate solutions we employ numerical techniques. In particular the boundary value problem (7) and (9) has been solved using the initial value or "shooting" method for 34 values of $B$ in $\frac{1}{2} \leqq B \leqq 100$. We describe the numerical method and present the results of the calculations here. Similar calculations were done for $B=1$ and 5 by J. C. Miller (see [1]).

For any fixed value of $B$ let $u=u(A, \theta)$ be the solution of the initial value problem

$$
\begin{aligned}
u \frac{d u}{d \theta} & =u B \sin ^{2} \theta-A \sin ^{4} \theta, \\
u\left(\frac{\pi}{2}\right) & =1,
\end{aligned}
$$

in the interval $-\pi / 2 \leqq \theta \leqq \pi / 2$. If the parameter $A=A(B)$ can be chosen such that

$$
\phi(A) \equiv u(A,-\pi / 2)-1=0,
$$

then it clearly follows that the function $y(\theta) \equiv u(A, \theta)$ is a solution of the boundary value problem (7) and (9) for the fixed pair of parameter values $B, A(B)$. To solve the transcendental equation (34) Newton's method is employed. That is, with some initial estimate $A_{0}$ of the root the iterates $A_{\nu}$ are defined by

$$
A_{\nu+1}=A_{\nu}-\frac{\phi\left(A_{\nu}\right)}{d \phi\left(A_{\nu}\right) / d A}, \quad \nu=0,1,2, \cdots .
$$


Since the solution $u(A, \theta)$ of (33) is continuously differentiable with respect to the parameter $A$ we may define

$$
v(A, \theta) \equiv \frac{\partial u(A, \theta)}{\partial A}
$$

and find from (33) that $v$ is the solution of the variational problem

$$
\begin{aligned}
\frac{d v}{d \theta} & =\left(\frac{A v}{u(A, \theta)}-1\right) \frac{\sin ^{4} \theta}{u(A, \theta)}, \\
v(\pi / 2) & =0 .
\end{aligned}
$$

It now follows from (34) that

$$
\frac{d \phi(A)}{d A}=v(A,-\pi / 2) .
$$

In the calculations the initial value problems (33) and (36) are integrated (from $\pi / 2$ to $-\pi / 2$ ) as a first order system using a Runge-Kutta method to start and the modified Adams predictor-corrector method to continue. The iterates (35) are evaluated using these computed solutions in (34) and (37). The iterations, for a given value of $B$, are terminated when $\left|A_{\nu+1}-A_{\nu}\right|<5 \times 10^{-6}$, which occurs after two or at most three steps, and the agreement is usually to eight digits. The converged value, $A_{v+1}$ $=A(B)$, is used as the initial estimate, $A_{0}$, of $A(B+\delta B)$. The increment $\delta B=10$ is used in $10 \leqq B \leqq 100$. The net spacing for the numerical integration is $\Delta \theta=\pi / 102$ for $B \leqq 12$ and $\Delta \theta=\pi / 502$ for $B \geqq 10$.

The thrust is computed by using Simpson's rule in (31). The singularity at $\theta=0$ is best treated by simply eliminating the two panels over $-\Delta \theta$ $\leqq \theta \leqq \Delta \theta$. This is consistent with the accuracy of Simpson's rule since for the exact solution

$$
y^{\mathrm{I}}(0)=y^{\mathrm{II}}(0)=y^{\mathrm{IV}}(0)=y^{\mathrm{V}}(0)=0, \quad y^{\mathrm{III}}(0)=2 B .
$$

The strange numbers of net points employed are specially chosen to avoid the need for end-corrections in the numerical integrations. The programming was very ably done by $\mathrm{W}$. H. Mitchell. The calculations were performed in about 50 seconds on an IBM 7094 at the W. H. Booth Computing Center at the California Institute of Technology.

Representative graphs of the solution $y(\theta)$ of (7) and (9) for several values of $B$ are shown in Fig. 3. The computed values of $A(B) / B$, $y(0)=p_{0} / p_{1}$ and $\bar{T}=T y(0) / p_{0} \sqrt{2 R h}$ are plotted in Fig. 4 . Values of $A(B) / B$ from the formula (14) are compared with results of the numerical solution in Table 1 . The agreement is quite good for all $B \geqq 10$ and the comparison at $B=100$ confirms the accuracy of the numerical work in a rather spectacular fashion. 


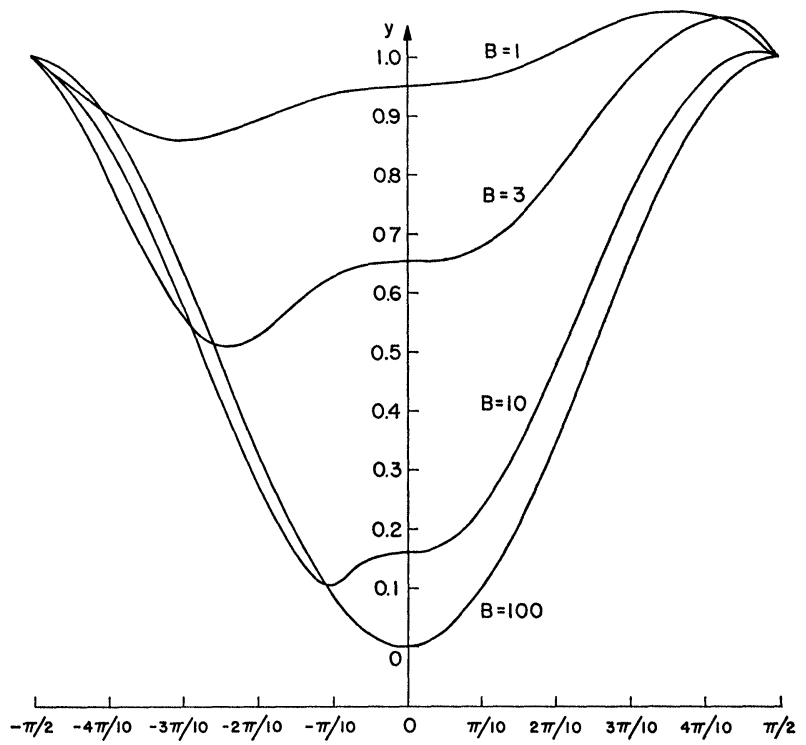

Fig. 3. $y(\theta)$ for various values of $B$

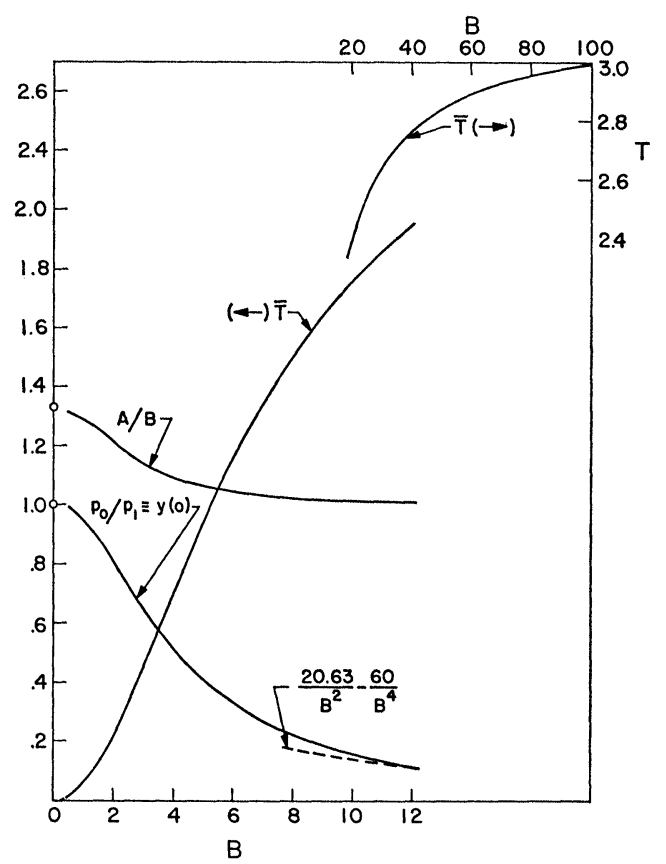

FIg. 4. Numerical results 
FLOW OF VISCOUS COMPRESSIBLE FLUID THROUGH A NARROW GAP 615

TABLE 1

Comparison of computed values of $A / B$ with those of formula (14)

\begin{tabular}{c|c|c|c|c}
\hline & $B$ & 5 & 10 & 100 \\
\cline { 2 - 5 } & \begin{tabular}{c|c}
$\left(1+2 / B^{2}-16 / B^{4}\right)$ \\
Computations
\end{tabular} & $\begin{array}{c}1.05400000 \\
1.06465511\end{array}$ & $\begin{array}{l}1.01840000 \\
1.01865676\end{array}$ & $\begin{array}{l}1.00019984 \\
1.00019988\end{array}$ \\
\hline
\end{tabular}

TABLE 2

Comparison of computed values of $y(0)$ with those of formula (38)

\begin{tabular}{|c|c|c|c|c|}
\hline & $B$ & 10 & 20 & 100 \\
\hline \multirow[t]{2}{*}{$y(0)$} & $\left(20.63 / B^{2}-600 / B^{4}\right)$ & 0.14600000 & 0.04782909 & 0.00205700 \\
\hline & Computations & 0.16164435 & 0.04811537 & 0.00205708 \\
\hline
\end{tabular}

An asymptotic formula for $y(0)=p_{0} / p_{1}$ can be obtained by using (22) and (25) in (19). This gives

$$
y(0) \sim \frac{20.63}{B^{2}}-\frac{600}{B^{4}}
$$

and its values are compared with those of the numerical work in Table 2. The agreement is not quite as good as in the previous case and very close agreement does not occur till $B>20$.

From (32) and the rough estimate following we obtain the crude formula for the thrust,

$$
\bar{T} \equiv \frac{T y(0)}{p_{0} \sqrt{2 R h}} \sim \pi\left(1-\frac{10}{B}\right) .
$$

However, this is not a very close estimate to any of the computed values in $B \leqq 100$. It is best at $B=100$, where we have the comparison:

$$
\begin{aligned}
\bar{T} \text { asym. } & =2.827, \\
\bar{T} \text { comps. } & =2.993 .
\end{aligned}
$$

Thus the formula (39) is just within $6 \%$ of the correct value at $B=100$ and we may expect that it (rapidly) improves for larger values of $B$.

4. Forced flow. In this case we must solve (1) subject to the boundary conditions (5), where $p_{2}<p_{0}$. For small $\beta$, power series of $p$ and $\alpha$ may again be employed. However, the lowest order term, corresponding to $\beta=0$ in (1), has independent significance since it represents the case of forced flow with a nonrotating cylinder. An integration yields in this case

$$
\left(\frac{p(\theta)}{p_{0}}\right)^{2}=1-\frac{\alpha}{2}\left[\frac{3}{2}\left(\phi+\frac{\pi}{2}\right)+\sin 2 \phi+\frac{\sin 4 \phi}{8}\right],
$$




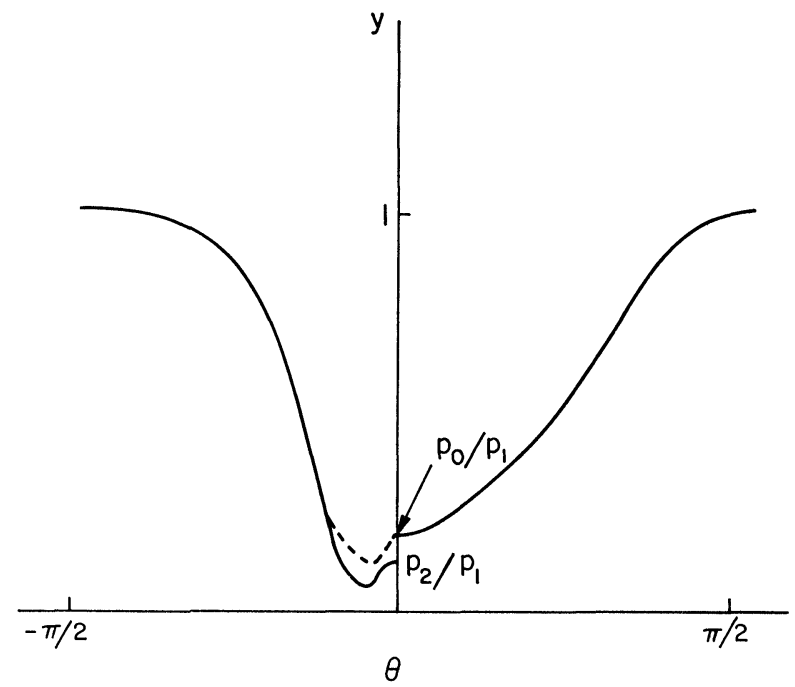

Fig. 5. Sketch of the solution of (7) for forced flow with rotating cylinder (i.e., $\beta>0$ )

and conditions (5) are satisfied if $\alpha$, which determines the mass flow rate, has the value

$$
\alpha=\frac{16}{3 \pi}\left[1-\left(\frac{p_{2}}{p_{0}}\right)^{2}\right] .
$$

With this solution the forced flow through a grating or lattice of stationary cylinders can be deduced in the manner described by J. B. Keller [3] for an incompressible fluid.

Continuing the perturbation procedure it is easy to write down the first and higher order corrections to the solution (40). However, the formulas are not very informative as the integrations cannot be carried out in terms of elementary functions.

The forced flow solution is not a periodic function of $\phi$ but we can still express the problem in terms of the variables $\theta=\phi-\pi / 2$ and $y(\theta)=p / p_{1}$. The boundary conditions (5) simply require that $y(\theta)$ be discontinuous at $\theta=0$ and satisfy there the jump condition

$$
y(0-)=\frac{p_{2}}{p_{0}} y(0+) .
$$

The solution $y(\theta)$ must satisfy the differential equation (7) in each of the intervals $\pi / 2>\theta>0$ and $0>\theta>-\pi / 2$, must take on the boundary values (9) and must satisfy the jump conditions (41). For the case of $p_{2} / p_{0}$ of order unity and $B \gg 1$ the singular perturbation solution is identical 
with that obtained previously except for numerically small negative values of $\theta$ (see Fig. 5). The reason is that the value of $A$ is determined to all inverse powers of $B$ by the outer solution. The value of $p_{0} / p_{1}=y(0+)$ is then found from the requirement that the inner expansion for $\tilde{\theta} \rightarrow+\infty$ match the outer expansion for $\theta \rightarrow 0+$. All inner expansions for $\tilde{\theta}<0$ will match the outer expansion, whatever the value of $p_{2} / p_{1}$. Thus the solutions for different values of $p_{2} / p_{0}$ have values of $A$ which differ only in transcendentally small terms, and the solutions are identical except when $\tilde{\theta}$ is finite and negative. Physically, this implies that for large $B$ the flow is independent of the exit pressure except right at the exit.

The previous numerical procedure is easily modified to treat the forced flow case. The first order system (33) and (36) is to be integrated from $\pi / 2$ to $\theta=0+$, then the jump conditions

$$
\begin{aligned}
& u(0-)=\frac{p_{2}}{p_{0}} u(0+), \\
& v(0-)=\frac{p_{2}}{p_{0}} v(0+)
\end{aligned}
$$

are applied and the integration continued from $\theta=0-$ to $-\pi / 2$. The rest of the calculations are unaltered.

\section{REFERENCES}

[1] G. I. Taylor and P. G. Saffman, Effects of compressibility at low Reynolds numbers, J. Aeronaut. Sci., 24 (1957), pp. 553-562.

[2] M. Reiner, Researches on the physics of air viscosity, Technical Report, Technion Research and Development Foundation, Haifa, Israel, 1956.

[3] J. B. KELLER, Viscous flow through a grating or lattice of cylinders, J. Fluid. Mech., 18 (1964), pp. 94-96. 